\title{
Paper Quality Aspects in Digital Broadcasting and Webcasting Systems: Bitrate versus Loudness
}

\author{
Przemysław Gilski, Sławomir Gajewski, and Jacek Stefański \\ Faculty of Electronics, Telecommunications and Informatics, Gdańsk University of Technology, Gdańsk, Poland
}

\begin{abstract}
In this paper the quality aspects of bitrate and loudness in digital broadcasting and webcasting systems are examined. The authors discuss a survey concerning user preferences related with processing and managing audio content. The coding efficiency of a popular audio format is analyzed in the context of storing media. An objective study on a representative group of signal samples, as well as a subjective study of the perceived quality of real-time broadcasted and webcasted radio programs are performed.
\end{abstract}

Keywords-broadcast technology, mobile communication, quality of experience, quality of service, webcast technology, wireless communication.

\section{Introduction}

Currently, digital broadcasting and webcasting systems are common in everyday life. They contribute to the spread and availability of information and educational resources, as well as entertainment.

The possibilities and limitations of existing technology lead to the search for new solutions that would satisfy user expectations. It is assumed, that high quality is closely linked with the bitrate assigned to a particular service. However, issues such as Quality of Service (QoS), a synonym for network performance and reliability, or Quality of Experience $(\mathrm{QoE})$, interpreted as the level of user acceptance, show that the quality of digital audio transmission systems can be interpreted in a different manner.

\section{Digital Audio Transmission}

At present, contemporary digital audio transmission services suffer strong competition from other broadcasting and non-broadcasting media, such as streaming platforms, also referred to as webcasting services, or various cloud-based storage platforms [1].

Broadcasting is a free-to-air service of consuming audio content. When users listen to music, terrestrial radio transmission occupies a predefined share of bandwidth and provides the same quality of the audio material for nearly all of them, regardless of the number of active users. An increase in the number of simultaneous users does not cause degradation in quality.
Webcasting services are undeniably a very popular mean of conveying audio content to the public. Their popularity is clearly visible in the number of dedicated applications available for popular mobile devices. However, in some cases the major drawback of webcasting services is their limited functionality and insufficient quality. Some service providers encourage users to purchase a premium account in order to consume high-quality content [2].

\section{Perceived User Quality}

The perceived user quality is a complex phenomenon, a mixture of the technical QoS and perceptual QoE. Due to the widespread and availability of mobile and portable devices, audio content can be consumed almost anytime and everywhere.

Broadcasters, webcasters and content providers must support a wide range of services with different codecs and bitrates to provide high-quality content under limited bandwidth conditions. In order to do so, they rely on tests concerning both subjective and objective quality metrics. In the subjective quality area, indisputably the most reliable method for quality assessment is via testing with a group of listeners. The most frequently used method is Mean Opinion Score (MOS) [3], where listeners rate the quality in a 5 -step scale from 1 (bad quality) to 5 (excellent quality). A newer 100-step scale methodology, called MUltiple Stimulus with Hidden Reference and Anchor (MUSHRA) [4], has also gained popularity.

In case of objective quality metrics testing is automated by software, which tries to predict the score that would be given by a human person. This way telecoms, manufacturers and content providers can easily evaluate the quality thorough the whole process of planning, implementation and maintenance of a particular product or service. A review of objective quality metrics can be found in [5].

\section{Quality Study}

The aim of this study was to examine the quality aspects of bitrate and loudness in digital broadcasting and webcasting systems. The study was performed on a group of 40 people aged between 20-25 years old; it consisted of four parts: 
1. Questionnaire performed in order to examine user habits and preferences when it comes to storing, processing and managing audio files.

2. Coding efficiency study of the most popular algorithm performed on a representative group of signal samples.

3. Objective quality assessment study performed on the same group of signal samples.

4. Subjective quality assessment study performed on real-time broadcasted and webcasted radio programs.

The experiment was conducted between January and February 2016, neither participant had hearing disorders.

\subsection{Questionnaire}

This part of the study was carried out in the form of a questionnaire consisting of three questions. It was performed prior to the listening tests, in order to learn user habits and expectations when it comes to downloading or streaming audio content. The results of this study are shown in Figs. 1-3.

According to obtained results, the majority prefers to use audio files processed using lossy compression algorithms.

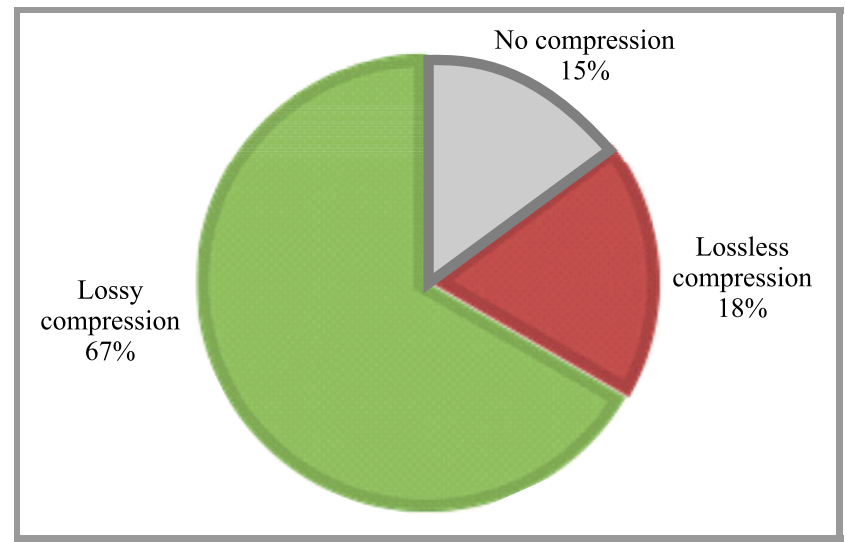

Fig. 1. Frequently chosen coding algorithm.

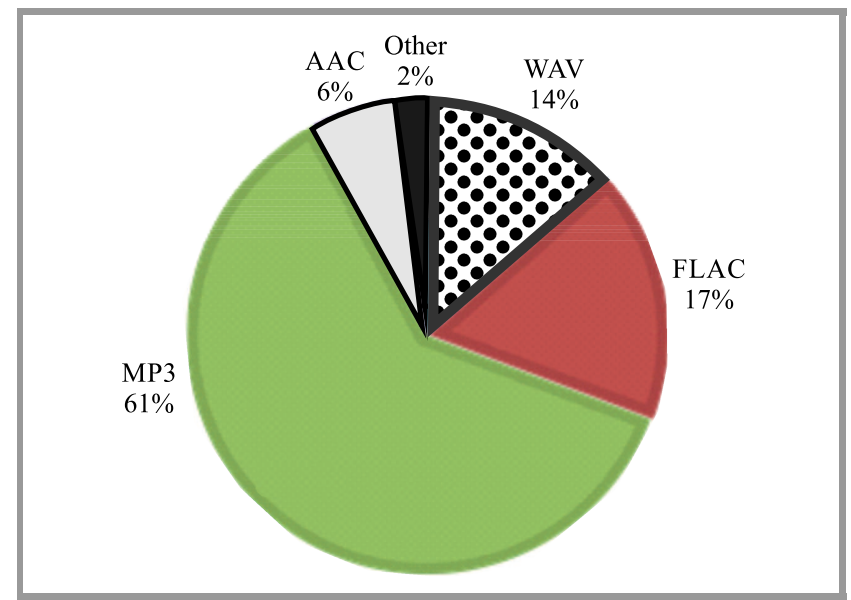

Fig. 2. Frequently chosen audio format.

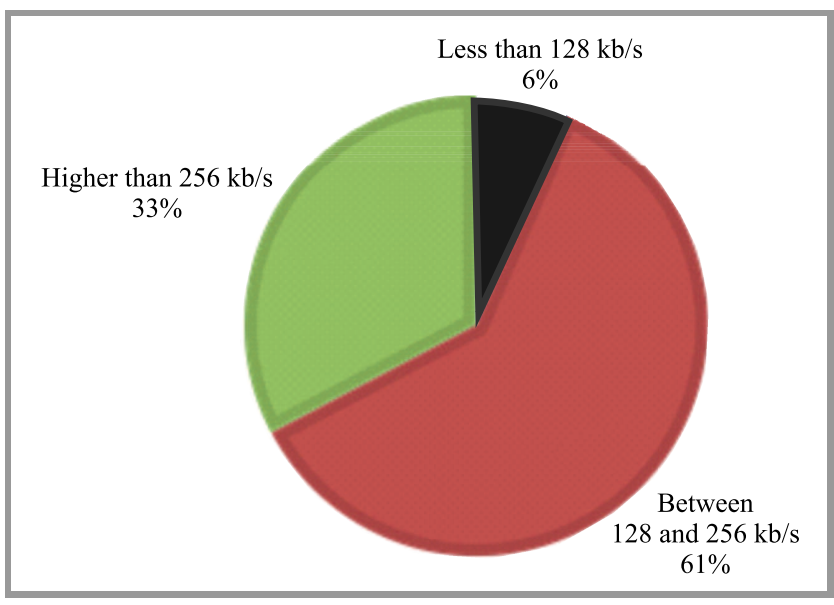

Fig. 3. Most frequently chosen bitrate.

About one third of them select audio materials that are either uncompressed or processed using lossless compression algorithms.

Surprisingly, with the vast development of new and more efficient coding algorithms, the MP3 is still the most popular audio format. Other formats such as Advanced Audio Coding (AAC), used in digital broadcasting systems such as Digital Audio Broadcasting plus (DAB+) [6], Free Lossless Audio Codec (FLAC) or WAVe audio format (WAV) gained 6,17 and $14 \%$ respectively.

Not surprisingly, whenever users have the opportunity, they favor the highest bitrate available. According to the study, more than a half selects a bitrate of $128-256 \mathrm{~kb} / \mathrm{s}$. One third prefers bitrates higher than $256 \mathrm{~kb} / \mathrm{s}$, whereas only $6 \%$ chooses a bitrate of less than $128 \mathrm{~kb} / \mathrm{s}$.

Of course, the quality of any digital service is strictly connected with the assigned bitrate. However, bandwidth is a very limited and costly resource, regardless whether talking about wired or wireless transmission. That is why low bitrates are mostly preferable by broadcasters and content providers, since they enable to introduce more services.

\subsection{Coding Efficiency}

When it comes to providing high quality content, especially at lower bitrates, one question arises - how much information could be lost? Audio coding and compression algorithms enable to shrink down the size of a file without seriously affecting the quality. Asides from lossy compression, every broadcast or webcast transmission causes additional degradation in quality. That is why scientists focus on developing new and efficient ways of processing the audio material, especially at low bitrates.

Based on the questionnaire, we decided to carry out a study concerning the coding efficiency of the most popular algorithm. A detailed description of the MP3 coding algorithm, in contrast with AAC, a popular algorithm utilized by many digital broadcasting and webcasting systems, can be found in [7].

The signal samples used during test have been divided into 3 categories: 
Table 1

Signal samples processed during test

\begin{tabular}{|c|c|c|c|}
\hline Category & File name & Description & Duration [s] \\
\hline \hline \multirow{3}{*}{ Speech and singing } & Female speech & Female lector in English & 23 \\
\cline { 2 - 4 } & Male speech & Male lector in English & 22 \\
\cline { 2 - 4 } & Quartet & Four voices (soprano, alto, tenor, bass) singing acapella & 28 \\
\hline \multirow{3}{*}{ Musical instruments } & Accordion & Accordion solo & 22 \\
\cline { 2 - 4 } & Trumpet & Trumpet solo & 32 \\
\cline { 2 - 4 } & Violin & Violin solo & 29 \\
\hline \multirow{2}{*}{ Popular music } & Billie Jean & Popular music piece & 27 \\
\cline { 2 - 4 } & Thriller & Popular music piece & 20 \\
\hline
\end{tabular}

1. Speech and singing - female and male speech, quartet.

2. Musical instruments - accordion, trumpet, violin.

3. Popular music - two music pieces by Michael Jackson.

Samples from category 1 and 2 were sourced from European Broadcast Union (EBU) [8], whereas those from category 3 came from the authors' private music library. The full list and description of signal samples used during tests is shown in Table 1. The sampling frequency of each file was set to $44.1 \mathrm{kHz}$.

The comparison between the size of the reference (original WAV file) and degraded (MP3 coded file) audio files, coded at different bitrates, is shown in Fig. 4.

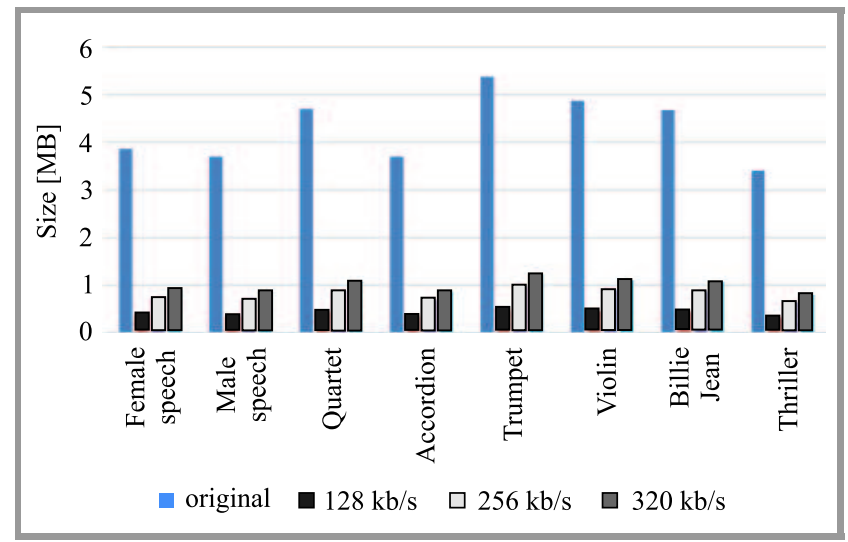

Fig. 4. Size of MP3 coded signal samples.

Of course, higher bitrates are strictly connected with better quality of the output signal. However, there is always a breakpoint, when managing or storing large and numerous files becomes a difficult and problematic task. As shown, lossy compression algorithms can lower the required storage space by tens of percent.

\subsection{Objective Test}

The same set of signal samples, as described in Table 1, was processed using an objective quality metric, called
ViSQOLAudio [9]. This algorithm compares the difference between the reference and degraded audio file. The sampling frequency of each original and processed file was set to $44.1 \mathrm{kHz}$. The result of this test, in MOS scale, is shown in Fig. 5.

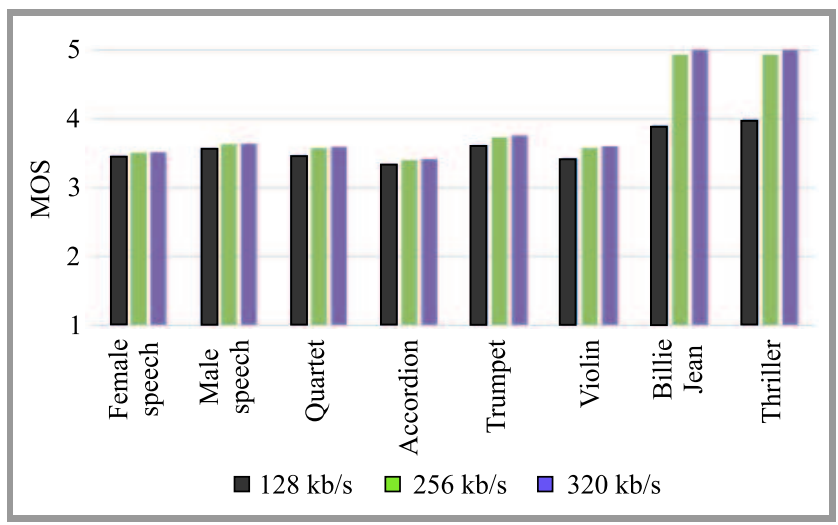

Fig. 5. Objective score of MP3 coded signal samples.

According to the study, the overall quality of the tested material was ranked as good (4 out of 5 points). In most cases, choosing a bitrate higher than $128 \mathrm{kbps}$ provides only a slight increase in quality.

As some scholars indicate, bitrates of approximately $256 \mathrm{~kb} / \mathrm{s}$ provide almost the same quality as the original unprocessed reference signal [10]. That is why one question arises - is it really necessary to design services that deliver audio content at bitrates higher than $128 \mathrm{~kb} / \mathrm{s}$, or even $256 \mathrm{~kb} / \mathrm{s}$.

\subsection{Subjective Test}

Subjective tests are regarded as expensive and time consuming. However, only users can give an accurate feedback about their expectations related with devices or content.

The aim of this part was to determine whether other aspects, besides mere assigned bitrate, i.e. the loudness level of the audio material, can affect the perceived quality. As 
it was notices during preparation, some broadcasters transmit audio content at higher volume than others. Therefore, this fact may be misleading, causing an individual to switch over to another radio program.

In general, webcasters transmit their content at lower bitrates than broadcasters, most often at $48 \mathrm{~kb} / \mathrm{s}$. That is why a group of 4 radio programs, available both terrestrial and online, has been chosen. The profile and assigned bitrate of each radio program is described in Table 2.

Table 2

Profile and assigned bitrate of broadcasted and webcasted radio programs

\begin{tabular}{|l|c|c|}
\hline $\begin{array}{c}\text { Radio program } \\
\text { profile }\end{array}$ & $\begin{array}{c}\text { Broadcast bitrate } \\
{[\mathrm{kb} / \mathrm{s}]}\end{array}$ & $\begin{array}{c}\text { Webcast bitrate } \\
{[\mathrm{kb} / \mathrm{s}]}\end{array}$ \\
\hline \hline Children & 72 & 48 \\
\hline Informative English & 64 & 48 \\
\hline Informative Polish & 64 & 48 \\
\hline Electronic music & 72 & 48 \\
\hline
\end{tabular}

Tests were carried out on two groups of listeners, 20 people in each, wearing AKG K550 closed-back headphones. Each participant assessed the quality individually and was not informed about the actual bitrate of the broadcasted or webcasted radio program.

The subjective tests were performed in turns, one listener after another, according to recommendation [11]. Each individual was asked to rank the overall quality of the same real-time transmitted radio program, both broadcasted and webcasted, in a 5-step MOS scale. They listened to each radio station for approximately 10-20 s.

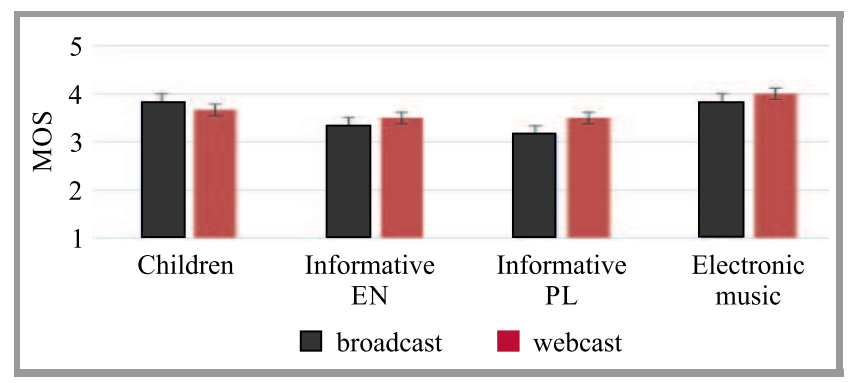

Fig. 6. Subjective scores - different volume.

The first group of 20 listeners was asked to assess the quality, with the webcasted material emitted at higher level of volume. The results of this study are shown in Fig. 6. Obtained subjective results have been processed

Table 3

ANOVA test results - different volume

\begin{tabular}{|l|c|c|c|c|}
\hline \multicolumn{1}{|c|}{ Category } & $\alpha$ & $P$ & $F_{\text {crit }}$ & $F$ \\
\hline \hline Broadcast & 0.05 & 0.59 & 3.09 & 0.65 \\
\hline Webcast & 0.05 & 0.75 & 3.09 & 0.41 \\
\hline
\end{tabular}

with the ANalysis Of VAriance (ANOVA) statistical analysis, as shown in Table 3 . The confidence interval was set to $95 \%$.

According to obtained results, in both cases the $P$ value was not less than $\alpha$. Additionally, the $F$ value did not exceed the $F_{\text {crit }}$.

Due to higher volume, the webcasted material, although transmitted at lower bitrate, was ranked not only as comparable with the broadcasted material, but even higher.

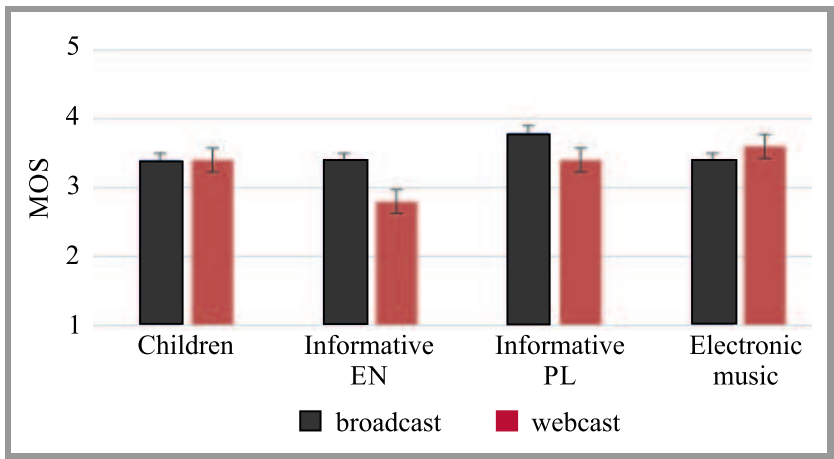

Fig. 7. Subjective scores - normalized volume.

The second group of 20 listeners was asked to rank the quality, where the loudness of both broadcasted and webcasted material was set to the same normalized level. The results of this tests are shown in Fig. 7. Obtained subjective results have been processed with the ANOVA statistical analysis, as shown in Table 4 . The confidence interval was set to $95 \%$.

Table 4

ANOVA test results - normalized volume

\begin{tabular}{|l|c|c|c|c|}
\hline Category & $\alpha$ & $P$ & $F_{\text {crit }}$ & $F$ \\
\hline \hline Broadcast & 0.05 & 0.85 & 3.24 & 0.26 \\
\hline Webcast & 0.05 & 0.45 & 3.24 & 0.92 \\
\hline
\end{tabular}

According to obtained results, in both cases the $P$ value was not less than $\alpha$. Additionally, the $F$ value did not exceed the $F_{\text {crit }}$.

It can be noticed, that when comparing audio material with the same loudness, the broadcasted radio programs were ranked as of higher quality. This observation can be made in real life, when various radio stations are transmitting programs at different level of volume.

Additionally, the current trend of mixing and mastering, especially popular music pieces, focuses on increasing the loudness level. Issues such as timbre, dynamics, space atmosphere, etc., come secondary [12].

\section{Conclusions}

Bandwidth is a very saturated and limited resource, regardless whether analyzing wired or wireless transmission. In the digital era, where users desire to consume high-quality 
content at any time and everywhere, quality plays a major role in the successful development and introduction of every new service.

The perceived quality is strictly connected with the assigned bitrate of the audio material. Not surprisingly, users prefer the highest bitrate whenever they have a possibility to make that choice. Companies realize this fact, sometimes offering premium quality only to users whom pay for their service. As it was proven, lossy compression algorithms can significantly decrease the required storage space for managing audio files. However, degraded audio files of $256 \mathrm{~kb} / \mathrm{s}$ and less may not always provide high quality, indistinguishable from the original file. Nevertheless, in most cases they assure good quality for the user.

According to obtained results, introducing broadcast or webcast audio material at bitrates of less than $128 \mathrm{~kb} / \mathrm{s}$ can provide users with content ranked between fair and good. This is an important issue, especially when talking about designing free or payable premium services.

Providing high quality content in the context of user QoE proved to be a complex phenomenon. As it was shown, the volume level of a radio program can also affect the perceived quality, as louder was ranked as better. This can cause uncertainty on the user side, causing him or her to switch to another louder station, which seems to be of better quality than the first stiller one.

It is vital to understand the pros and cons of different technologies. Both QoS and QoE are very crucial factors when it comes to managing bandwidth resources. Consumers will welcome every new service or product that offers new possibilities and can meet their demands.

\section{References}

[1] P. Gilski and R. J. Katulski, "Electronic media in the life of a modern man”, Elektronika - konstrukcje, technologie, zastosowania, vol. 3, pp. 7-11, 2016 (in Polish).

[2] P. Gilski and J. Stefański, "Digital audio broadcasting or webcasting: a network quality perspective", J. of Telecommun. and Inform. Technol., no. 1, pp. 9-15, 2016.

[3] "Methods for the subjective assessment of small impairments in audio systems including multichannel sound systems", ITU Recom. BS.1116-1, 1997.

[4] "Method for the subjective assessment of intermediate quality level of coding systems", ITU Recom. BS.1534-1, 2003.

[5] S. Möller et al., "Speech quality estimation: models and trends", IEEE Sig. Proces. Mag., vol. 28, pp. 18-28, 2011.

[6] "Digital Audio Broadcasting (DAB); Transport of Advanced Audio Coding (AAC) audio", ETSI Technical Specification 102 563, 2010.

[7] K. Brandenburg, "MP3 and AAC explained", in Proc. AES 17th Int. Conf. on High Quality Audio Coding, Florence, Italy, 1999, pp. 1-12.

[8] "Sound Quality Assessment Material recordings for subjective tests (SQUAM CD)", EBU Technical Document 3253, 2008.

[9] A. Hines et al., "ViSQOLAudio: An objective audio quality metric for low bitrate codecs", J. of the Acoust. Soc. of America, vol. 137, pp. 449-455, 2015.

[10] A. Hines et al., "Perceived audio quality for streaming stereo music", in Proc. 22nd ACM Int. Conf. on Multimedia MM'14, Orlando, FL, USA, 2014, pp. 1173-1176.
[11] "General methods for the subjective assessment of sound quality", ITU Recom. BS.1284, 2003.

[12] E. Vickers, "The loudness war: do louder, hypercompressed recordings sell better?", J. of the Audio Engin. Soc., vol. 59, no. 5, pp. 346-351, 2011.

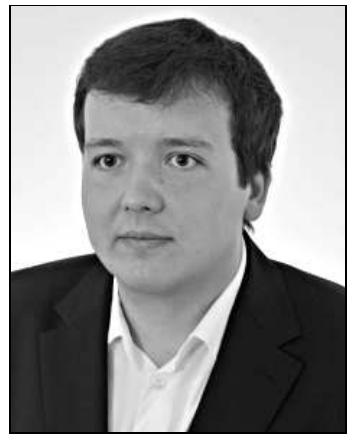

Przemysław Gilski received his B.Sc. and M.Sc. degrees in Telecommunications Engineering from Gdańsk University of Technology (GUT), Poland, in 2012 and 2013, respectively. Currently he is a Ph.D. student at the Department of Radio Communication Systems and Networks (DRCSN), GUT. His research and development interests include digital video and audio broadcasting systems, software-defined radio technology, location services and radio navigation systems, as well as quality measurements in mobile networks.

E-mail: pgilski@eti.pg.gda.pl

Faculty of Electronics, Telecommunications and Informatics

Department of Radio Communication Systems and Networks

Gdańsk University of Technology

Gabriela Narutowicza st 11/12

80-233 Gdańsk, Poland

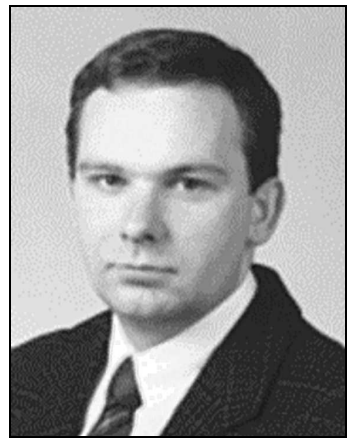

Sławomir Gajewski is a Professor at the Department of Radio Communication Systems and Networks, Gdańsk University of Technology. His research and development interests include analysis, simulation, design and measurements of radio communication systems, spectrum spreading, OFDM transmission, signal processing, software radio design, radio resource utilization and management in modern cellular systems, resource reuse, interference and load management, throughput and capacity optimization in cellular and broadcasting systems, radio network planning and optimization, maritime radio communication systems design, and intelligent transport systems.

E-mail: slagaj@eti.pg.gda.pl

Faculty of Electronics, Telecommunications and Informatics

Department of Radio Communication Systems

and Networks

Gdańsk University of Technology

Gabriela Narutowicza st 11/12

80-233 Gdańsk, Poland 


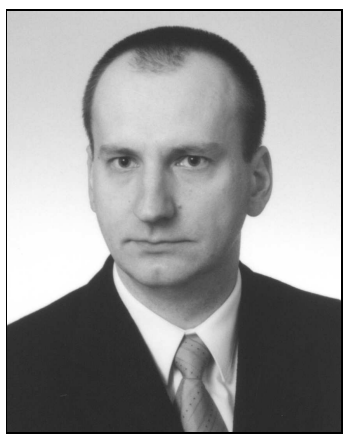

Jacek Stefański received his M.Sc., Ph.D. and D.Sc. degrees in Telecommunications Engineering from Gdańsk University of Technology (GUT), Poland, in 1993, 2000 and 2012, respectively. From 1993 to 2000 he worked as an Assistant Professor at the Department of Radio Communication Systems and Networks (DRCSN), GUT.

Since 2001 he has been working as an associate professor at the DRCSN. His research and development interests include analysis, simulation, design and measurements of cellular, wireless and trunked radio systems, techniques of digital modulation, channel coding, signal spreading, radio signal reception, measurement of radio wave propaga- tion, field strength prediction, software radio design, location services, ad-hoc sensor networks, radio monitoring systems and radio navigation systems. He is the author and co-author of more than 250 papers. He is a member of the Electromagnetic Compatibility Section of the Electronics and Telecommunications Committee, Polish Academy of Science and the Institute of Electrical and Electronics Engineers organization.

E-mail: jstef@eti.pg.gda.pl Faculty of Electronics, Telecommunications and Informatics

Department of Radio Communication Systems and Networks

Gdańsk University of Technology

Gabriela Narutowicza st 11/12

80-233 Gdańsk, Poland 